\title{
Understanding nutrition transition among internal rural-to-urban migrant women in Nairobi, Kenya
}

\author{
R. Peters, H. Elsey, J.N. Newell and A.J. Hill \\ Leeds Institute of Health Sciences, University of Leeds, Leeds, LS2 9NL.
}

Kenya is undergoing a nutrition transition as the population increasingly consumes foods that are high in fat, energy dense, and processed. Furthermore, overweight and obesity rates are on the rise, especially among women ( $45 \%$ of whom were overweight or obese in 2016) ${ }^{(1,2)}$. This nutrition transition appears to be particularly affecting the higher socio-economic groups. However, the urban poorest are also vulnerable. One of the key underlying determinants of this transition is rapid urbanisation ${ }^{(3)}$. In Nairobi, around $75 \%$ of the population currently reside in slums, and the total number of people residing in slums is rapidly increasing due to rural-urban migration ${ }^{(4)}$. Our study examined the perceptions and experiences of rural-to-urban migrant women on their current urban food environment and changes in dietary behaviour. The aim was to better understand the mechanisms behind the adoption of obesogenic dietary behaviour in context of rapid urbanisation.

Our study combined in-depth individual interviews, focus group discussions and PhotoVoice in three of Nairobi's slums: Korogocho; Viwandani and Kibera. Migrant women $(n=75)$ were purposefully sampled based on the following criteria: being aged 18-49 years, previously living in a rural area before migrating to Nairobi's slums, and various other socio-economic and demographic criteria. The interviews used a Kiswahili semi-structured interview guide. The interviews were transcribed verbatim and analysed using a thematic framework in NVivo version 11 (QSR International).

The most substantial change the migrant women experienced (after rural-urban migration) was the switch from a farming/trading system to a cash-based food system. Key influences on the adoption of obesogenic dietary behaviour included lack of a steady income and lack of time/means to cook at home. Furthermore, the preferences of participants' children seemed to play a major role in the consumption of new energy-dense foods such as chapatti and fries as they become aware of near neighbours' cooking and the taste of roadside foods. The diversification to energy-dense roadside food consumption was strongly encouraged by participants' physical food environment as they preferred to buy foods at the roadside since they were nearby and accessible. Respondents perceived the availability of traditional vegetables to be low, and the local market to be far away, while bad hygienic growing and selling conditions also inhibited vegetable consumption. Finally, slum neighbourhood-specific norms and values within each slum neighbourhood seemed to influence perceptions of desired body image and adoption of food preparation methods ("when you go to visit people they cook like that, so you have to adapt to cook like that."). These influences seemed to stimulate their use of fatty, pre-cooked energydense foods, reducing their consumption of traditional vegetables and rural cereals, while changing their food preparation methods from boiling to frying with cooking oil.

The rural-to-urban migrants' attitudes towards these changes in diet and food environment were mixed. Many women were unhappy as they perceived the urban foods to be unhealthy and they were not able to access the traditional foods. In contrast, being able to change foods daily and consume meat and fish were viewed as positive changes.

Our study highlights the need to adopt a multilevel approach and involve the community in participatory decision-making and intervention design to effectively prevent obesogenic dietary behaviour among these women. It demonstrates that rural-urban migration has the potential to be detrimental to health as the respondents in this study featured strong characteristics of the nutrition transition. Further research is needed to assess the generalizability of the findings.

1. Steyn NP \& McHiza ZJ. (2014) Ann N Y Acad Sci 1311, 88-101.

2. WHO Global Infobase (2016) Available at: http://apps.who.int/gho/data/node.main.A896?lang=en.

3. Ziraba AK, Fotso JC \& Ochako R (2009) BMC Public Health 9, 465.

4. Zulu EM, Beguy D et al. (2011) J Urban Health 88(2), 185-99. 